\title{
Irreversible desiccation shrinkage of cement paste caused by cement grain dissolution and hydrate precipitation
}

\author{
Xiaodan Li • Zachary C. Grasley (iD · Jeffrey W. Bullard • \\ Edward J. Garboczi
}

Received: 14 June 2016/ Accepted: 16 November 2016/Published online: 23 November 2016

(C) RILEM 2016

\begin{abstract}
When cementitious materials are dried, internal stresses are generated that lead to desiccation shrinkage, a portion of which is irreversible. Previous research has indicated that, while a cementitious composite is subjected to a state of stress, dissolution of cement grains and precipitation of hydrates can yield irreversible creep strains, and it is hypothesized that the same process can lead to irreversible shrinkage during drying. To evaluate this hypothesis, a computationally implemented model integrating a microstructural evolution model with a finite element calculation routine was utilized. This computationally implemented model is capable of predicting the magnitude of shrinkage deformation of cement paste during drying conditions
\end{abstract}

X. Li · Z. C. Grasley ( ()

Zachry Department of Civil Engineering, Texas A\&M

University, College Station, TX, USA

e-mail:xiaodanl@tamu.edu

Z. C. Grasley

e-mail: zgrasley@tamu.edu

\section{J. W. Bullard}

Materials and Structural Systems Division, Engineering Laboratory, National Institute of Standards and

Technology, Gaithersburg, MD, USA

e-mail: jeffrey.bullard@nist.gov

\section{E. J. Garboczi}

Applied Chemicals and Materials Division, Material Measurement Laboratory, National Institute of Standards and Technology, Boulder, CO, USA

e-mail: edward.garboczi@ nist.gov as a result of cement grain dissolution and hydrate precipitation. From the simulation results, the mechanism of cement grain dissolution and hydrate precipitation can lead to significant shrinkage behavior of cement paste, and it is also a potential mechanism resulting in the irreversible component of desiccation shrinkage at early ages (e.g., while the hydration rate is significant). The predicted irreversible shrinkage decreases with the age at which drying is initiated as a result of the decreasing hydration reaction rate.

Keywords Microstructure $\cdot$ Shrinkage $\cdot$ Cement paste $\cdot$ Modeling $\cdot$ Dissolution

\section{Introduction}

Desiccation shrinkage occurs as a result of pore water loss inside cement paste, and is a time-dependent, characteristic behavior of cementitious materials. Both external drying (e.g., through moisture diffusion and evaporation) and internal drying (e.g., when capillary pore water is consumed during hydration or other chemical reactions) (see e.g., [1-3]) can induce desiccation shrinkage. At high internal relative humidity $(\mathrm{RH})$, the origin of desiccation shrinkage has been primarily attributed to the change in the pore fluid pressure, which can lead to corresponding compression of the surrounding solid microstructure [4-8]. The mechanical response to this compression is experimentally measured as desiccation shrinkage. An important observation is that even under constant RH (which 
indicates constant pore fluid pressure), shrinkage would continuously occur, producing an irreversible component of deformation and a creep-like behavior of cement paste desiccation shrinkage [9]. Thus, in this paper, irreversible shrinkage is defined as the shrinkage strains that will not ultimately be recovered upon returning the internal RH to the level that preceded any desiccation (i.e., the internal RH at full saturation).

Despite devoted efforts to investigate the mechanisms behind shrinkage, no unified theory has been derived to explain the creep and irreversible components of desiccation shrinkage. It is generally believed that microstructural and chemical changes are the origin of these irreversible deformations, and many theories regarding these changes have been proposed, such as the formation of bonds between pressed surfaces [6], further formation of hydration products [10], creation of new interlayer space [5] and dissolution of adsorbent molecules [11]. The major components that are generally attributed as responsible for the irreversible strains are the amorphous calcium silicate hydrate $(\mathrm{C}-\mathrm{S}-\mathrm{H})$ [3, 12, 13] and distributed pores inside cement paste $[12,14]$. A change in the internal $\mathrm{RH}$ can lead to an alteration in the internal structure of the C-S-H gel, manifesting as a dryingaccelerated "aging" process of the $\mathrm{C}-\mathrm{S}-\mathrm{H}$, potentially leading to an irreversible component of deformation. Besides the mechanisms that are directly related to these fundamental microstructure changes, internal microcracking in cement paste is another potential mechanism of irreversible desiccation shrinkage [3].

A computational scheme which couples the hydration microstructural model thermodynamic hydration and microstructure evolution (THAMES) $[15,16]$ and a time-stepping finite element calculation model has been recently developed [17, 18]. This computational scheme is capable of predicting the long-term, timedependent mechanical properties of cement paste as a function of time-evolving microstructures. One strength of this model is that it can separate different deformation components and study their effects individually. According to simulation results from the computational scheme, stress redistribution associated with the dissolution of solid load-bearing constituents can lead to significant irreversible, creep or relaxation of cement paste when the microstructure is subjected to external loads [17-19]. In support of these simulation results indicating that creep can result from dissolution of load-bearing constituents, Pignatelli et al. [20] recently showed that the tendencies for creep and dissolution of $\mathrm{C}-\mathrm{S}-\mathrm{H}$ are closely related. Furthermore, experiments have indicated a significant reduction in creep rate at low $\mathrm{RH}$ where dissolution of cement grains and other phases would be hindered (see, e.g., [21-24]).

In previous studies, the authors focused on the stress state change as a result of dissolution occurring while under external mechanical loadings; however, internal pore fluid pressure changes (due to e.g., desiccation) can also contribute to the stress state within cement paste, and this latter influence was not considered in previous papers. Thus, a hypothesis is proposed that the dissolution of cement grain particles and precipitation of hydrates during the hydration process can cause early-age, irreversible desiccation shrinkage. To evaluate this hypothesis and quantify the significance of this mechanism, the aforementioned computational scheme was utilized in this paper to simulate the early-age desiccation shrinkage behavior of cement paste. From the simulation results, two major mechanisms that can lead to desiccation shrinkage were studied and compared in this paper: (1) instantaneous elastic deformation of cement paste solid phases as a result of pore fluid pressure change and (2) the progressive deformation due to concurrent cement grain dissolution during the hydration reaction. Preliminary simulation results of cement paste shrinkage were presented in [25], and based on these simulation results, a complete set of virtual experiments were carried out in this paper to simulate cyclic drying and rewetting conditions to give a more direct investigation of the mechanisms behind irreversible shrinkage. Additionally, besides the study of the mechanism of cement grain dissolution and hydrate precipitation, the effect of $\mathrm{C}-\mathrm{S}-\mathrm{H}$ viscoplasticity in leading to irreversible shrinkage is also analyzed. As poroelasticity has been widely utilized to model desiccation shrinkage of porous materials such as cement paste, the simulation results are also compared to simple poroelastic calculations.

\section{Computationally implemented model}

\subsection{Microstructure model and finite element analysis}

The computationally implemented model involves a hydration microstructural model THAMES and a finite element analysis model. THAMES is capable 
of generating the three dimensional (3D) time-evolving microstructures of cement paste during the hydration process at the micrometer level $[15,16]$. With the initial input of experimentally measured clinker constituents' mass fractions, the particle size distribution of cement grains and the water to cement ratio $(w /$ c) at the sub-particle level, THAMES calculates the amount of dissolved cement grains at different ages. The reaction rate of clinkers with water follows the empirically-derived clinker reaction rate equations strictly (Parrot and Killoh [26]), and the dissolution process of cement grain particles are determined by the phenomenological dissolution kinetics [15]. Based on the mass of dissolved cement grains into the pore fluid, THAMES calculates the pore fluid elemental composition at each age. This elemental composition is then input into a thermodynamic engine Gibbs energy minimization (GEMS) [27, 28], which has been implemented in THAMES, to calculate the mass of precipitated hydrates while assuming a near-equilibrium state is reached between the hydration products and pore fluid solution. A more detailed description of THAMES can be found in $[15,17,18]$.

As desiccation shrinkage is a mechanical response to an internal state of stress, prediction of such deformation requires mechanical analysis. With the input of a series of 3D images of simulated cement paste from THAMES (each of which contains $100^{3}$ voxels with the dimension of each voxel of $1 \mu \mathrm{m}^{3}$ ) at different ages, a finite element analysis routine is used to calculate the internal stress/strain fields of cement paste at each microstructure age. The voxels in the 3D microstructures are meshed in the finite element program utilizing a spatially aligned numerical discretization [29], and in this way, each voxel becomes an eight node trilinear cubic finite element consisting of one single phase. Elastic mechanical properties (e.g., Young's modulus and Poisson's ratio) are assigned to all the voxels. Through minimizing the total mechanical energy stored in the composite under strain-controlled periodic conditions, the stress/strain fields inside cement paste can be calculated $[17,18]$. The predicted external stress under the applied strain is the volume-averaged stress over the whole composite. With the knowledge of any two of the linearly elastic properties, the other elastic, mechanical moduli of the composite can be calculated through solving the elastic equations on a regular finite element mesh [30].
One thing to note in the program is that, to account for the dissolution process of cement grains and precipitation process of hydrates, a major assumption is made in the finite element routine. It is assumed that, once phase dissolution occurs inside one voxel, stress redistribution occurs simultaneously and instantaneously from this dissolved voxel to the surrounding solid phase voxels to satisfy linear momentum conservation. The newly formed phases will form in a stressfree state within the deformed configuration (or voxel) and carry no memories of the historical responses of this voxel. In this way, "apparent strain" is produced inside the composite as a result of the dissolutionprecipitation process. Here, the term "apparent strain" is defined as the strain that is produced in the voxel that exhibits phase change between the time of mechanical loading and the time right before the formation of a given solid in the voxel. Since the voxel must be stressfree at the moment of formation, the apparent strain is strain that does not have a corresponding stress in the voxel. From a continuum mechanics perspective, the apparent strain can be treated in the same fashion as other "free" or "eigen" strains (e.g., strain induced by temperature change or moisture state change). Constitutive viscoelastic/viscoplastic (VE/VP) behavior of cement paste at the macroscale can happen as a result of the stress redistribution process associated with the load-bearing solids dissolution even when all the microscopic phases inside the program are purely elastic. The term "apparent VE/VP" is reserved in this paper to describe the VE/VP behavior of cement paste that occurs strictly due to the dissolution process while disregarding the inherent VE or VP properties of phases (e.g., C-S-H). More detailed procedures and theoretical derivations of the computational model may be found in [17-19].

\subsection{Shrinkage prediction}

\subsubsection{New computationally implemented model}

The current version of the computational scheme has an important limitation in that the mechanical responses of the microstructure are predicted under strain-controlled periodic boundary conditions, and currently there is no effective way to switch the boundary conditions from controlled strain to controlled stress. As the time-evolving desiccation shrinkage strain of cement paste is normally measured under 
zero external stress (e.g., traction free boundaries) and negative internal pore fluid pressure, to simulate this experimental measurement environment, Boltzmann's superposition principle [31, 32] was used. Figure 1 shows the flow chart for calculating the overall shrinkage strain of cement paste utilizing the computational program and the superposition principle.

To simulate experiments of desiccation shrinkage measurement and to predict the evolution of the magnitude of shrinkage strain with time, four major steps were conducted in the virtual, computational experiment. First, time was discretized into $n$ different time steps, with each time step representing one particular age. For the simulations, a stepwise negative pore pressure $H(t) p_{0}$ (where $H(t)$ is the Heaviside function) and zero external periodic boundary strain were applied on a list of microstructures that were generated by THAMES for all drying ages $t_{1}, t_{2}, t_{3}, \ldots$, $t_{n}$ (which means that pore pressure was applied on cement paste starting at time $t_{1}, t_{2}, t_{3}, \ldots, t_{n}$ and each time $t_{i}$ is the age represented by time step $i$ ). The negative pore pressure was applied to the fluid phases of the simulated microstructures for the purpose of simulating the drying process, and the evolution of the stress fields of the whole composite as a result of the assigned pore fluid pressure were predicted by the computational program.

The spatially averaged hydrostatic stress of the composite as a function of time for different drying ages was calculated from the simulation results. The hydrostatic stress of the composite per unit uniform pore pressure was determined as

$\sigma_{t_{m}}^{\text {unit }}\left(t_{i}\right)=\sigma_{t_{m}}^{p_{0}}\left(t_{i}\right) / p_{0}$,

where $\sigma_{t_{m}}^{\text {unit }}\left(t_{i}\right)$ is the value of the hydrostatic stress at time $t_{i}$ per unit pore pressure when dried at age $t_{m}$, $\sigma_{t_{m}}^{p_{0}}\left(t_{i}\right)$ is the hydrostatic stress at time $t_{i}$ under pore pressure $p_{0}$ when dried at age $t_{m}, m=1,2,3, \ldots, n$ and $i=1,2,3, \ldots, n$. The resultant hydrostatic stress history of the composite under any given internal pore pressure history, $p(t)$, may be determined according to

$\sigma\left(t_{i}\right)=\sum_{k=m}^{i}\left\{\sigma_{t_{k}}^{u n i t}\left(t_{i}\right)\left[p\left(t_{k}\right)-p\left(t_{k-1}\right)\right]\right\}$,

where $\sigma\left(t_{i}\right)$ is the hydrostatic stress of the composite at time $t_{i}$ under the given pore pressure history.

The desiccation shrinkage strain was calculated utilizing the hydrostatic stress history of the composite from Eq. (2) and the evolving VE/VP properties of the composite simulated using the computationally implemented model as described in [17, 18]. In order to perform the shrinkage calculation, it was necessary to apply a virtual boundary hydrostatic stress history of$\sigma\left(t_{i}\right)$ to maintain an overall spatially averaged zero stress state (corresponding to "free shrinkage"), as shown in Fig. 1. Then, the strain history that must necessarily be applied at each time step to achieve- $\sigma\left(t_{i}\right)$ was calculated according to the superposition principle via

$$
\begin{aligned}
& \text { if } i=1, \quad \varepsilon\left(t_{i}\right)=\frac{-\sigma\left(t_{i}\right)}{3 K_{t_{i}}\left(t_{i}\right)} \\
& \text { if } i \neq 1, \quad \varepsilon\left(t_{i}\right)=\frac{-\sigma\left(t_{i}\right)-\sum_{k=1}^{i-1}\left[\varepsilon\left(t_{k}\right) K_{t_{k}}\left(t_{i}\right)\right]}{3 K_{t_{i}}\left(t_{i}\right)},
\end{aligned}
$$

where $\varepsilon\left(t_{i}\right)$ is the additional linear strain to be applied on the composite at time $t_{i}$ to achieve the desired boundary stress history, and $K_{t_{k}}\left(t_{i}\right)$ is the apparent VE/ VP bulk modulus (i.e., computed from the model in $[17,18])$ of the composite at time $t_{i}$ when loaded at age $t_{k}$. More explicitly, the apparent VE/VP bulk modulus is computed from the simulated dilatational stress
Fig. 1 Flow chart for shrinkage strain calculation under strain-controlled boundary conditions

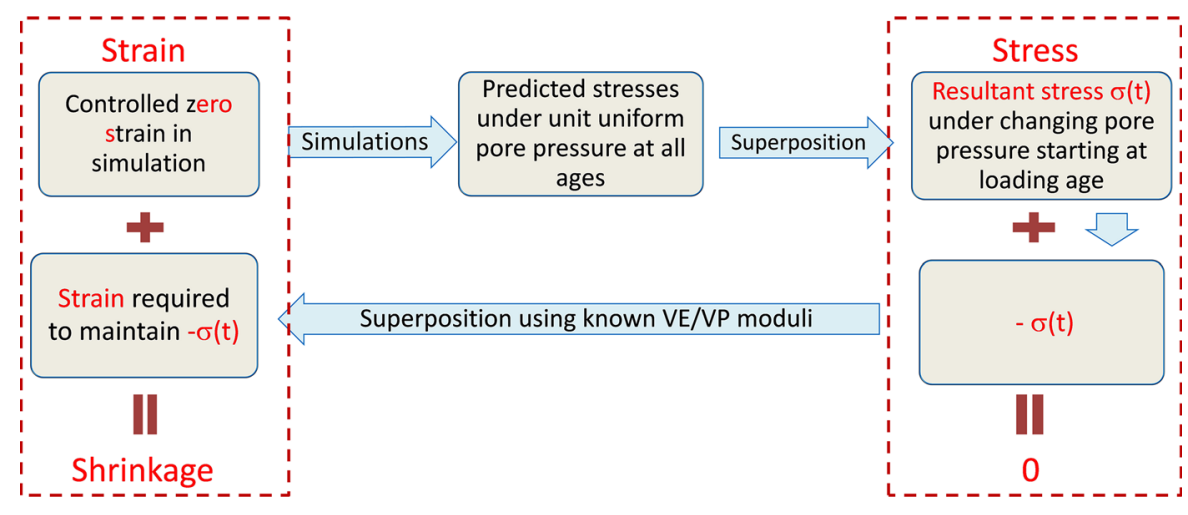


relaxation (owing to dissolution of cement grains) for a composite subjected to a periodic dilatational strain. The resultant linear shrinkage strain, $\varepsilon_{\text {shrinkage }}\left(t_{i}\right)$, of the composite is calculated as

$\varepsilon_{\text {shrinkage }}\left(t_{i}\right)=\sum_{k=1}^{i} \varepsilon\left(t_{k}\right)$.

In this way, the predicted time-evolving shrinkage strain occurs due to the concomitant elastic deformation of the solid microstructure responding to the pore fluid pressure changes as well as the dissolution of cement grains and subsequent stress redistributions in the microstructure. Although Eq. (1) through Eq. (4) were intended for calculating the time-evolved shrinkage strain of cement paste during the drying process, if a specimen is exposed to re-wetting and the internal $\mathrm{RH}$ of the simulated cement paste increases as a function of time, then the time-dependent swelling strain of cement paste may be predicted following the same procedures, except that the final results obtained would have an opposite sign, denoting negative shrinkage (i.e., expansion). The ability to simulate re-wetting allows direct evaluation of reversibility and irreversibility of the simulated desiccation shrinkage.

\subsubsection{Classic poroelastic model}

Among analytical models for predicting the desiccation shrinkage of cementitious materials, from a mechanics perspective, poroelasticity has been used to predict the elastic, recoverable shrinkage of cement paste $[1,9,33,34]$. A poroelastic expression for the change in linear shrinkage strain may be written as

$$
\mathrm{d} \varepsilon_{\mathrm{sh}}(t)=\frac{S(t) d p(t)+p(t) d S(t)}{3}\left(\frac{1}{K_{p}(t)}-\frac{1}{K_{s}}\right),
$$

where $t$ denotes time, $\varepsilon_{\mathrm{sh}}(t)$ is the time-dependent function of linear shrinkage strain, $S(t)$ is the function of saturation coefficient (fraction of pores filled with liquid pore solution), $p(t)$ is the pore fluid pressure, $K_{p}(t)$ is the age-dependent function of bulk modulus of the drained porous medium and $K_{s}$ is the bulk modulus of the solid skeleton, which can be considered as constant. Comparing to $K_{t_{k}}\left(t_{i}\right)$ in Eq. (3), $K_{p}(t)$ here is the age dependent elastic instantaneous bulk modulus of the porous medium, while $K_{t_{k}}\left(t_{i}\right)$ is a continuous function denoting the time-dependent apparent VE/VP properties of the VE/VP material that includes relaxation associated with dissolution. At loading age $t_{\text {load }}, K_{t_{\text {load }}}(0)=K_{p}\left(t_{\text {load }}\right)$. As it is instructive toward understanding the role of dissolution on desiccation shrinkage, subsequent sections include comparisons between the computationally predicted shrinkage and the poroelastic shrinkage determined from (5).

\section{Simulation results and discussions}

Microstructures of hydrating cement composites at different ages (from $1 \mathrm{~d}$ to $56 \mathrm{~d}$ ) were examined in this paper to predict the time-dependent shrinkage strain of cement pastes. Unless stated otherwise, all the phases inside cement pastes were approximated to be linearly elastic with elastic properties taken from [35]. Using the power of the model to examine only one deformation mechanism at a time, here the predicted irreversible shrinkage occurs strictly due to the time-dependent dissolution of load-bearing cement grains.

\subsection{Different ages of desiccation initiation}

The desiccation process induces negative pore pressure in the pores of cement pastes. When a step function pore fluid pressure history is applied to cement paste pore structures, instantaneous, elastic shrinkage will occur at the time of the instantaneous application of pore pressure. All of the simulated timedependent increases in shrinkage strain after the instantaneous application of the pore pressure were associated strictly with cement grain dissolution. The time dependent shrinkage due to phase dissolution is irreversible due to the precipitation of new hydration products that 'lock in' the microstructure in the deformed state. Time dependent hydrate precipitation (which increases the composite stiffness) also ensures that only a portion of the initial elastic shrinkage deformation upon the step pore pressure loading is reversible. Figure 2 shows the predicted, normalized linear shrinkage strain of $0.40 \mathrm{w} / \mathrm{c}$ cement paste at ages of desiccation initiation of 1 and 4 days, where the shrinkage strain is normalized by the magnitude of applied pore pressure. It should be noted that the decay in the degree of pore liquid saturation in the simulated microstructures (which are generated by THAMES simulating the hydrating cement paste under sealed 
conditions) is due strictly to self-desiccation effects. Thus, when predicting the precise shrinkage strain of a cement composite, the normalized strain cannot be directly scaled with changes in pore pressure since such changes are always associated with changes in the degree of pore saturation. Figure 2 (and later Fig. 3) is strictly meant to illustrate how shrinkage may yet increase even at constant pore pressure, but is not meant to be a predictive tool.

From Fig. 2, cement pastes establish elastic deformation under applied pore pressure. As the stiffness of older specimens is greater than for younger specimens, older specimens are less compliant to internal and external stress state changes, and this yields overall less elastic deformation (or instantaneous shrinkage in this case). After the initial application of internal stresses, cement pastes continue to shrink under constant pore pressure, leading to substantial irreversible shrinkage due to cement grain dissolution and hydrate precipitation. Meanwhile, under the applied constant pore fluid pressure, a later drying initiation age will lead to smaller shrinkage strain increase, as well as slower shrinkage rate. One major reason behind the predicted smaller and slower shrinkage in older specimens is that the hydration reaction rate of cement paste decreases with age (and thus also the dissolution rate of cement grains), and this could lead to less irreversibility in predicted shrinkage. Another potential reason leading to decreasing shrinkage rate with time is that, when specimens are older, less water is present inside cement paste due to water

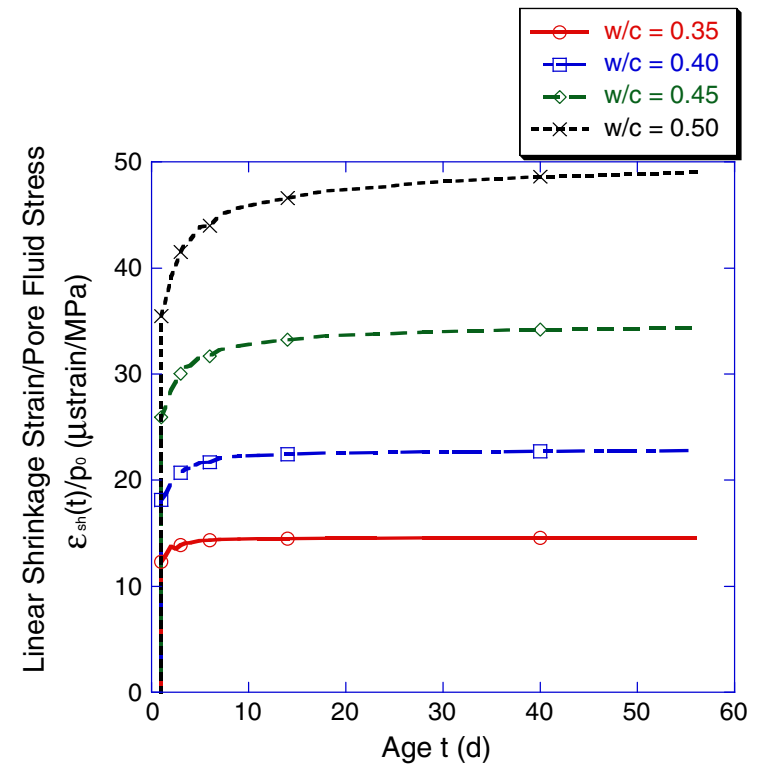

Fig. 3 Shrinkage strain normalized by the applied pore fluid pressure for different w/c at a drying initiation age of 1 day. The time dependent shrinkage strain was considered to occur strictly due to dissolution of load-bearing cement grains

consumption in the hydration reaction, resulting in a smaller overall contraction of the solid skeleton because the pore fluid pressure is exerted within a smaller fraction of the composite volume. Thus, based on the simulation results and aforementioned reasons, the irreversible shrinkage occurring due to cement grain dissolution and hydrate precipitation is likely to decrease with age, and this agrees with the
Fig. 2 Shrinkage strain normalized by the applied pore fluid pressure in a desiccating $0.40 \mathrm{w} / \mathrm{c}$ cement paste with drying initiated at different ages ( 1 and 4 days). In this graph, time dependent shrinkage strain was considered to occur strictly due to the dissolution of load-bearing cement grains

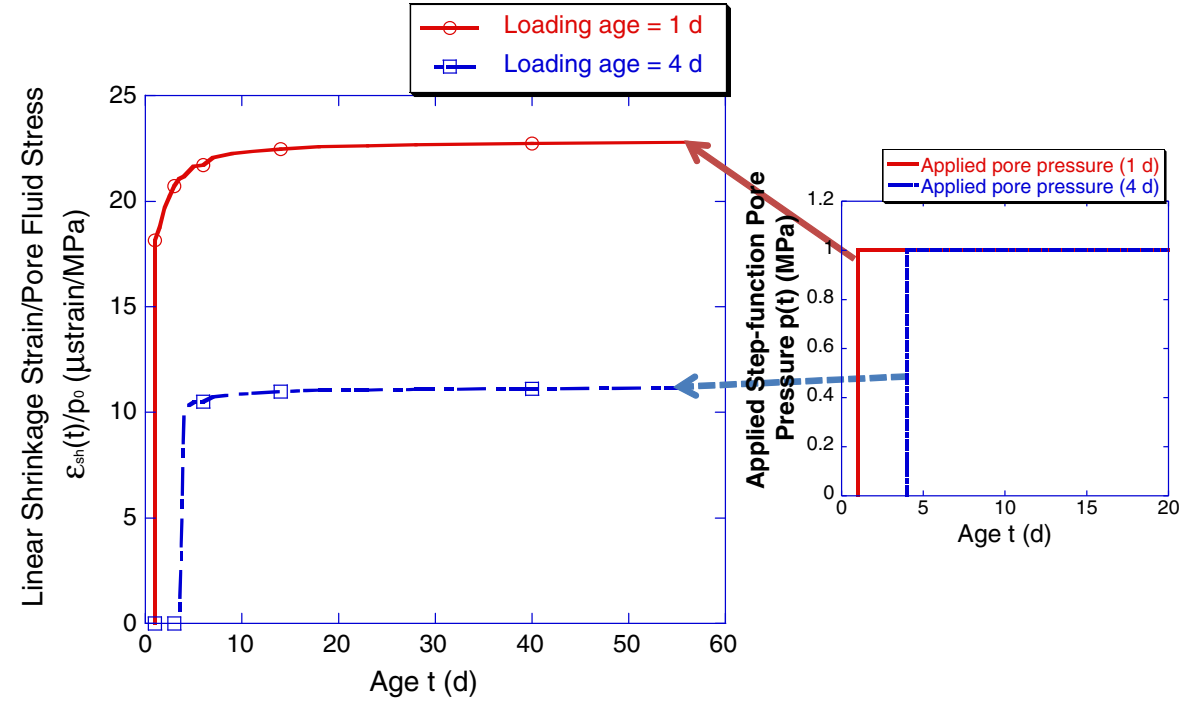


experimental data [36]. Furthermore, recent experimental results indicate that purely poroelastic models (which neglect dissolution effects) provide reasonably accurate predictions of mortar shrinkage for very mature samples where ongoing hydration is insignificant [37].

\subsection{Different w/c}

Figure 3 shows the predicted, normalized linear shrinkage strain for cement pastes with different $w / c$ from 0.35 to 0.50 with drying initiating at 1 day, where the shrinkage strain is normalized by the magnitude of stepapplied pore fluid pressure. From Fig. 3, it can be seen that, for the same pore pressure step, lower $w / c$ leads to slower shrinkage rate and smaller shrinkage magnitude than higher $w / c$ due to its lower water content and higher stiffness. The shrinkage rate of cement pastes for all $w / c$ is predicted to decrease with age.

\subsection{Time-evolving RH}

In the previous simulations (i.e., Figs. 2, 3), stepfunction pore fluid pressure histories were applied to cement pastes. In real life, the pore fluid pressure inside cement paste highly depends on the internal RH and changes with age. The relationship between the pore fluid pressure and internal RH can be approximated through the combined Kelvin-Laplace equation as

$p_{l}-p_{g}=\ln \left(\frac{\mathrm{RH}}{\mathrm{RH}_{0}}\right) \frac{R T}{v_{w}}$,

where $p_{l}$ is the pore liquid pressure and $p_{g}$ is the vapor pressure that exists in equilibrium above a flat surface or pore liquid. As $p_{g} \ll p_{l}$ over the range of pressures that induce shrinkage, $p_{g}$ will henceforth be neglected. $\mathrm{RH}$ is the current internal relative humidity, $\mathrm{RH}_{0}$ is the reference configuration internal relative humidity, ${ }^{1}$

\footnotetext{
${ }^{1}$ The initial RH (i.e., prior to any desiccation) in cement-based materials is suppressed by the reduction in water activity caused by the presence of dissolved species in the pore fluid [38]. Such effects can be accounted for as in [39], whereby the dissolved species and their concentrations are explicitly accounted for in a modified version of Eq. (6). An alternative approach is taken here whereby one defines $\mathrm{RH}_{0}$ not as $\mathrm{RH}=100 \%$ (i.e., the $\mathrm{RH}$ that is measured over a flat surface of pure water), but rather as the relative humidity above a flat surface of the pore solution as it exists prior to any desiccation. The latter approach is taken here, using experimental data from the literature.
}

$R$ is the universal gas constant, $v_{w}$ is the molar volume of water, and $T$ is the temperature in Kelvin [40]. In Eq. (6), one simplification is made that the effect of time dependent changes in the dissolved species concentration on the chemical potential of the pore fluid is neglected. A modified version of Eq. (6) should be used if one wishes to simulate evolving chemistry of the pore solution during desiccation [39].

To simulate realistic shrinkage behavior of cement paste utilizing the computational program, realistic time-evolving pore pressure or RH history inside cement paste is required. THAMES predicts the full microstructure evolution including the emptying of pores due to self-desiccation. Thus, THAMES is able to indicate which voxels (i.e., those filled with pore water) should be subjected to pore pressure changes for shrinkage modeling. However, THAMES does not resolve the nanometric pore sizes that would be needed to calculate the magnitude of the pore pressures. Therefore, rather than simulating the pore pressure history directly, measured internal $\mathrm{RH}$ data were utilized from experiments carried out where a small sensor was embedded in a self-desiccating (sealed from external drying) mortar bar [41]. Since the referenced experiments only involved mortars rather than pastes and since the cement utilized in the specimens was not characterized to the extent necessary to utilize THAMES to simulate the microstructure evolution, it was not deemed useful to compare the actual measured autogenous shrinkage of the mortar bar with simulated autogenous shrinkage of the paste. The RH history was simply utilized to represent a "typical" history for simulation purposes. With the input of this empirically measured internal RH history, the time-evolving pore fluid pressure can be calculated and the shrinkage strain as a result of the timedependent RH history as well as hydration reaction process can be predicted by the program.

Figure 4 shows the predicted time-dependent shrinkage strain of $0.40 \mathrm{w} / \mathrm{c}$ cement paste. The aforementioned experimentally measured internal RH data [41] from a self-desiccating mortar with the same $w / c$ as the simulated microstructure was used to quantify the evolving pore pressure history through (6), which was input into the program. The temperature of the simulation matched that of the experiment (298 K).

One thing to note is that, in reality, the rate of hydration reaction has a close relationship with the 
internal RH of cement paste. At low to intermediate $\mathrm{RH}$, the hydration reaction significantly slows down [42] or even stops due to changes in the chemical potential of the pore water and thus the thermodynamic equilibrium state [38]. Since the simulations presented in this paper were all conducted under relatively high $\mathrm{RH}$ conditions (with $\mathrm{RH}>80 \%$ ), it was assumed, for simplification, that the hydration rate was independent of the internal RH. While experiments [43] have indicated that hydration rates may be reduced $20-60 \%$ at $90 \%$ internal $\mathrm{RH}$ versus the fully water saturated condition, accounting for such a reduction in hydration rate would not change the magnitudes of the model predictions - it would only change the time required to achieve the predicted magnitudes. In fact, in the simulations involving purely elastic phases, as the predicted shrinkage strain by the computational scheme is strictly related to the degree of hydration instead of the hydration reaction rate, the shrinkage versus $\mathrm{RH}$ relationship is unique and independent of the hydration rate.

In Fig. 4, besides the time-evolving measured $\mathrm{RH}$ history on the right hand $Y$ axis, which leads to timeevolving pore fluid pressure, three different sets of predicted shrinkage strain history are included. The solid line and the dashed shrinkage strain line with square markers were obtained from the computationally predicted data. To obtain the dashed shrinkage strain line with square markers, all the phases inside each cement paste microstructure at all ages were considered purely elastic, and the deformation of each voxel was independent of the microstructures from other ages, or in other words, stress/strain history independent. This implies that the shrinkage strain shown in this dashed square marked line occurs purely as a result of the elastic response of cement paste to pore fluid pressure increase. Different drying initiation ages or historical microstructure evolutions will not affect the generation of this line. The solid shrinkage strain line, on the other hand, besides the elastic response of cement paste to pore fluid pressure changes, also considers the effects of microstructure evolution. Thus, the effect of stress redistribution and free strain generation that occurs during the cement grain dissolution and hydrate precipitation process are included in the generation of this solid line, resulting in a higher prediction of the desiccation shrinkage strain versus the purely elastic prediction. The differences between these two lines are the predicted irreversible shrinkage that occurs due to cement grain dissolution and hydrate precipitation.

The dashed shrinkage line with diamond markers in Fig. 4 was obtained from the poroelastic calculation results utilizing a discretized form of Eq. (5) in the computational model,

$$
\varepsilon_{\mathrm{sh}}\left(t_{i}\right)=\sum_{i=1}^{n} \frac{S\left(t_{i}\right) \Delta p\left(t_{i}\right)+p\left(t_{i}\right) \Delta S\left(t_{i}\right)}{3}\left(\frac{1}{K_{p}\left(t_{i}\right)}-\frac{1}{K_{s}}\right),
$$

where $\Delta p\left(t_{i}\right)=p\left(t_{i}\right)-p\left(t_{i-1}\right)$, denoting the pore pressure difference between two different time steps. The degree of saturation $S\left(t_{i}\right)$ was calculated utilizing the microstructures generated by THAMES, with $S(t)=\frac{\text { Number of pores filled with water }}{\text { Total number of pores }}$. The shrinkage predicted by poroelasticity follows the same trend as the predicted elastic recoverable shrinkage (the dashed line with square markers). One potential reason to explain the differences between the two dashed shrinkage prediction lines is that, for a partially saturated porous material, poroelasticity has significant drawbacks, and its accuracy highly depends on the microstructure (pore size distribution) of the material [34]. From the comparison between the solid shrinkage line and the two dashed shrinkage lines, dissolution of load-bearing cement grains and precipitation of stress-free hydrates during the hydration and shrinkage process, which occur simultaneously, is a significant mechanism leading to irreversible shrinkage. Poroelasticity underestimates desiccation shrinkage, as has been noted previously [1,9]. However, while Lura et al. [9] and Grasley and Leung [1] suggested that inherent viscoelasticity or creep of the cement paste phases might account for the underprediction, here we suggest that the internal stress redistribution associated with cement grain dissolution plays a role in such under-prediction at early ages. As cement paste ages during drying, the difference between the poroelastic calculation and the overall computational prediction (including both dissolution effect and pore pressure change) increases, as can be seen in Fig. 4.

\subsection{Recovering $\mathrm{RH}$}

Using the same RH data as used in Fig. 4 but forcing RH to return back to its initial value after 6 (Fig. 5a) 


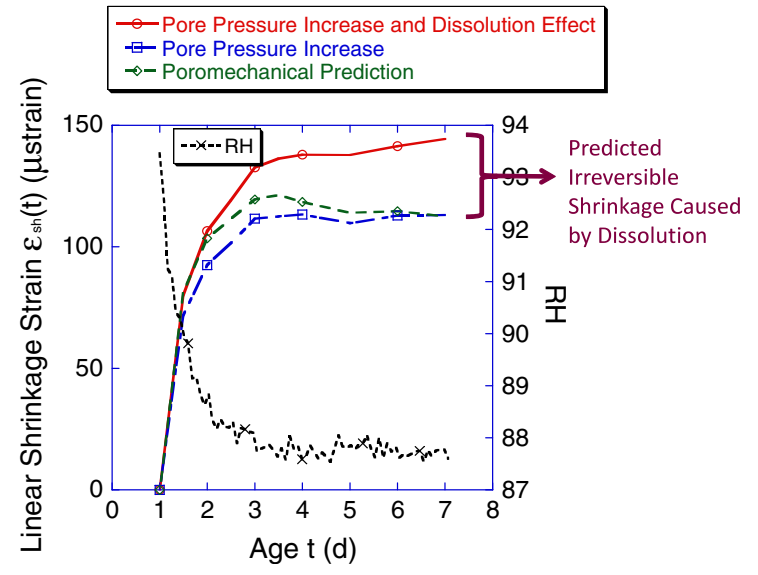

Fig. 4 Predicted shrinkage strain of $0.40 \mathrm{w} / \mathrm{c}$ cement paste with drying initiated at age of 1 day. In this graph, the computationally predicted data is shown (i) by treating cement paste at all ages as a microstructure-dependent time-evolving material (solid line), which shows the overall shrinkage due to both pore pressure increase and hydration reaction effects; (ii) by treating cement paste as purely elastic while neglecting phase dissolution effects (dashed shrinkage line with square markers), which shows the shrinkage of cement paste occurring purely due to instantaneous elastic deformation; and (iii) by poroelasticity (dashed shrinkage line with diamond markers). The RH history (dotted line with cross markers) was also included in this graph on the right hand $Y$ axis

and 7.5 days (Fig. 5b) of drying, the results shown in Fig. 5 can be obtained for drying initiating at 1 and 3.5 days. Microstructures of $0.40 \mathrm{w} / \mathrm{c}$ cement paste were used in the simulations, and the temperature was kept constant at $293 \mathrm{~K}$. In both Fig. 5a, b, the RH histories were included on the right hand $Y$ axis. The solid shrinkage lines show the overall shrinkage due to both pore pressure change and cement grain dissolution/ hydration precipitation effects, and the dashed shrinkage lines show the predicted shrinkage of cement paste occurring purely due to pore pressure change. In both figures, cement paste shrinks with decreasing RH and swells with recovering RH. After RH returns back to its initial value, the irreversible shrinkage strains caused by the dissolution/hydrate precipitation effect could be predicted by the differences between the solid lines and the dashed lines. Because THAMES does not resolve nanometer sized pores, it is unable to define the sorption isotherm for the microstructure and thus unable to predict the increase in the degree of pore saturation with increases in RH. Therefore, the increase in pore saturation level upon increases in $\mathrm{RH}$ is neglected in the simulations involving (a)
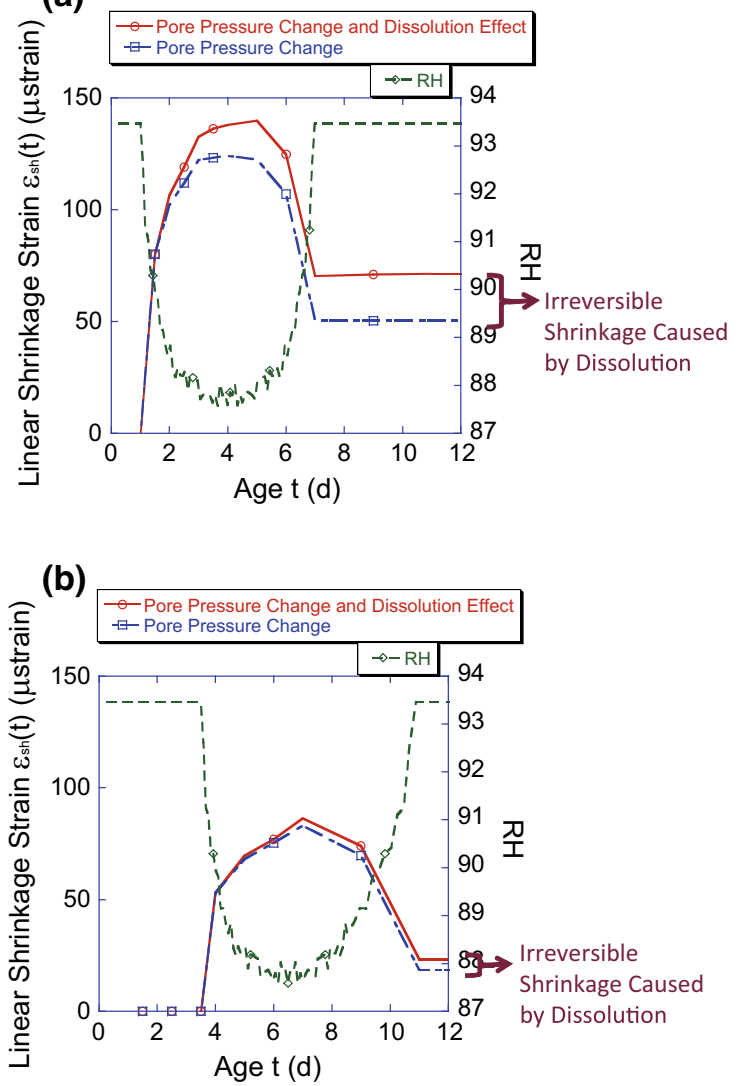

Fig. 5 Predicted shrinkage strain of $0.40 \mathrm{w} / \mathrm{c}$ cement paste with drying initiated at ages of $\mathbf{a} 1$ day and $\mathbf{b} 3.5$ days. In both graphs, the solid lines show the overall shrinkage due to both pore pressure change and hydration reaction effects, while the dashed shrinkage lines show the recoverable shrinkage of cement paste occurring purely due to pore pressure change. Internal $\mathrm{RH}$ history was also included in this graph on the right hand $Y$ axis

rewetting; this leads to slight under prediction of the irreversibility of the predicted shrinkages (as more water in the pores would lead to pressure being exerted over a greater fraction of the microstructure).

Comparing to Fig. 5b, Fig. 5a shows the shrinkage strain history of cement paste when drying initiated at an earlier age, and it can be seen that, when dried at earlier ages, a much larger irreversible shrinkage would be observed due to the more active hydration reaction at earlier ages (and thus higher dissolution and precipitation rates). These results suggest that steps to increase the degree of hydration prior to drying initiation (e.g., wet curing and heat curing) would reduce the irreversible component of desiccation shrinkage. Note that countering the effect of 
increased degree of hydration on reducing irreversible shrinkage is the additional shrinkage that may occur as a result of the refined pore structure associated with wet curing [44].

In the model simulations, after the RH returns back to its initial value or pore pressure returns back to zero, an irreversible shrinkage exists as a result of the increasing stiffness in the cement paste. At earlier ages, cement paste shrinks as pore pressure increases, while at later ages, when pore pressure returns back to zero, cement paste would not swell as much because of the increased stiffness, leaving an irreversible shrinkage value shown in Fig. 5.

\subsection{Cyclic drying and rewetting}

In Fig. 6, a cyclic drying and rewetting process was applied on $0.45 \mathrm{w} / \mathrm{c}$ cement paste at different ages of drying initiation of 1 and 2 days. The RH history applied in Fig. 6 follows the function

$R H(t)=100-10[1-\cos (2 \pi \cdot f \cdot t)](\%)$,

where $\mathrm{RH}(t)$ is the internal RH history as a function of time, $t$ represents the time in days after the start of drying, and $f$ denotes the frequency of the cyclic drying and rewetting. In this way, $\mathrm{RH}$ becomes a sinusoidal function oscillating between 100 and $80 \%$ with each cycle period equal to $1 / f$ in days. According to Eqs. (6) and (8), the internal pore pressure also becomes a sinusoidal function starting from zero pore fluid stress and oscillates with the frequency of $f$. According to the simulated shrinkage strain shown in Fig. 6, which includes both the elastic deformation of cement paste due to pore pressure changes and cement grain dissolution/hydrate precipitation effects, the irreversible strain increases with age under cyclic drying and rewetting and can be directly observed at the time when pore pressure returns back to zero, revealed in Fig. 6 as the strains at the bottoms of the oscillating curves. As discussed in the previous sections, younger specimens would yield a larger ultimate irreversible shrinkage, and the simulated maximum strain under maximum pore pressure per drying cycle decreases with age as a result of increasing stiffness of the cement paste. The irreversible component of desiccation shrinkage associated with cement grain dissolution and hydrate precipitation theoretically achieves an ultimate value as indicated above if one presumes the solid phases are
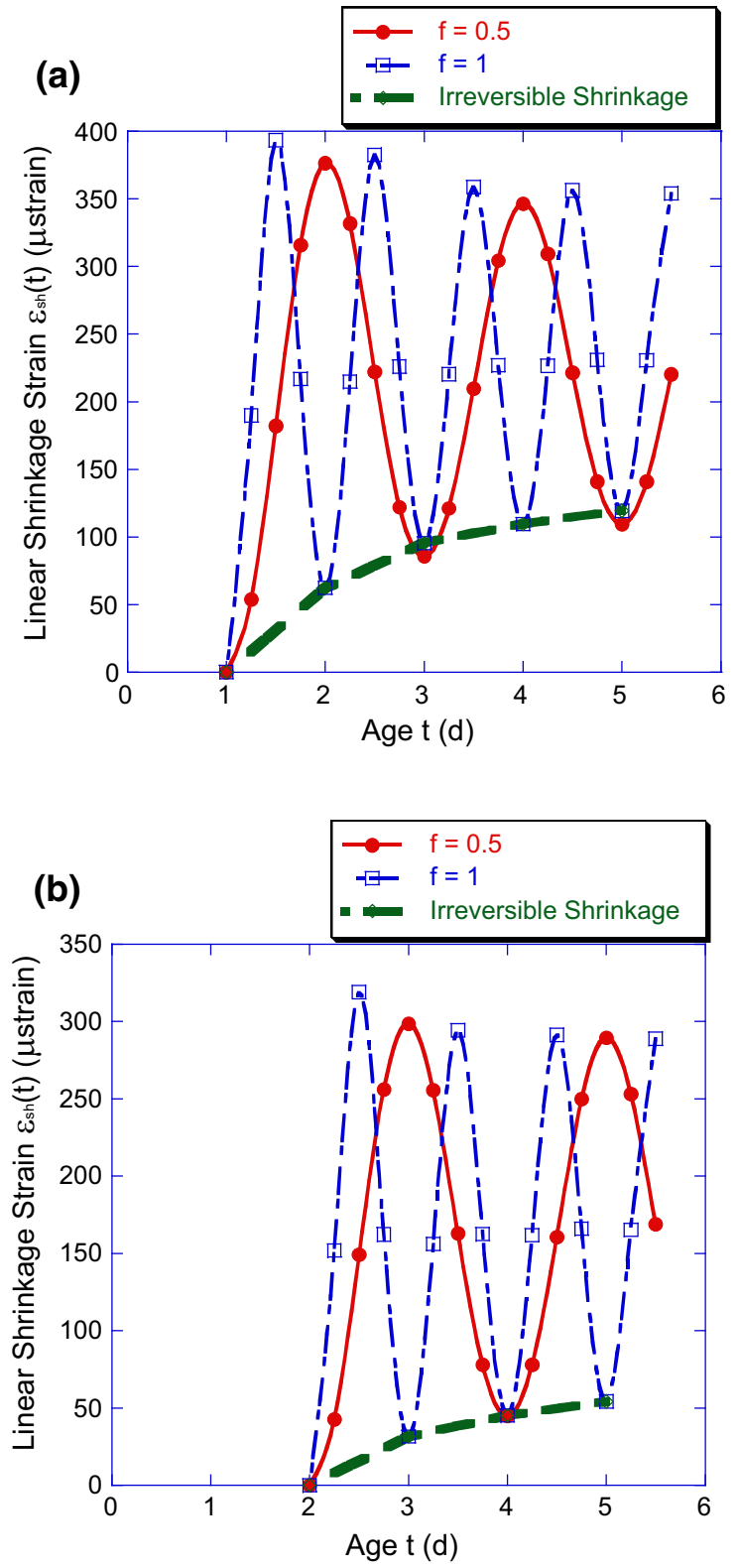

Fig. 6 Predicted shrinkage strain of 0.45 w/c cement paste when exposed to cyclic drying with different frequencies $(f=0.5$ and $f=1)$ at the initial drying ages of a 1 day and b 2 days. All the lines in this figure show the predicted shrinkage as a result of cyclic drying due to both pore pressure change and cement grain dissolution/hydrate precipitation effects

strictly elastic. This ultimate value would depend on several parameters, including the porosity, pore size distribution, phases present and their respective elastic properties, the drying and hydration kinetics, and the degree of hydration at the onset of desiccation. Given 
the complexity and multi-parameter dependency of this ultimate irreversible shrinkage, it is not plausible to derive a simple analytical expression to calculate this value.

According to Fig. 6a, more drying and rewetting cycles under the same time period would slightly increase the irreversible shrinkage value, but this increase is less than $10 \%$. It is generally accepted that, under cyclic drying and rewetting, most irreversible shrinkage occurs in the first cycle of drying and rewetting, and the shrinkage strain in the following cycles can be treated as reversible shrinkage [3, 45], but at early ages, according to the simulation results, irreversible shrinkage is more age-dependent rather than cycle-dependent. If the period of one drying cycle is significantly long enough (e.g., 5 days in Fig. 6a), the shrinkage occurring after the first cycle can be treated as reversible, but if the period of one drying and wetting cycle is relatively short, the irreversible shrinkage occurring after the first cycle is not negligible.

\subsection{Combined irreversibility due to dissolution and intrinsic plasticity}

As noted in the introduction, irreversible desiccation shrinkage has often been attributed to irreversible deformation of the $\mathrm{C}-\mathrm{S}-\mathrm{H}$ phase. Here, we evaluate the combined irreversible deformation associated with both irreversible deformation of the $\mathrm{C}-\mathrm{S}-\mathrm{H}$ and cement grain dissolution/hydrate precipitation effects. The quasi-instantaneous irreversible deformation of concrete associated with quasi-instantaneous application of stress is generally attributed to microcracking rather than plasticity of the phases (see, e.g., the discussion in $[3,46,47]$ and the references therein). Thus, $\mathrm{C}-\mathrm{S}-\mathrm{H}$ is modeled to exhibit both an instantaneous elastic response and a time-dependent viscoplastic (VP) response to stress.

When utilizing the same RH history as in Fig. 6 and treating the $\mathrm{C}-\mathrm{S}-\mathrm{H}$ phases inside cement paste as VP, Fig. 7 can be obtained showing the shrinkage occurring due to pore pressure changes, cement grain dissolution effects and $\mathrm{C}-\mathrm{S}-\mathrm{H}$ viscoplasticity. As there are no sufficient data currently available for modeling the relaxation of the $\mathrm{C}-\mathrm{S}-\mathrm{H}$ phase over several days due to experimental challenges, for simulation purposes, in Fig. 7, the VP Young's modulus of $\mathrm{C}-\mathrm{S}-\mathrm{H}$ was set to be $E(t)=$
11.2 GPa $+11.2 \exp (-0.2 t) \mathrm{GPa}$, and the Poisson's ratio of $\mathrm{C}-\mathrm{S}-\mathrm{H}$ was assumed to have a constant value of 0.25 . The relaxation function chosen for $\mathrm{C}-\mathrm{S}-\mathrm{H}$ is based on the instantaneous elastic Young's modulus reported in Haecker et al. [35], $E(0)=22.4 \mathrm{GPa}$, with an assumed $50 \%$ ultimate relaxation. There are little data available in the literature to guide the modeling of $\mathrm{C}-\mathrm{S}-\mathrm{H}$ relaxation. However, as the purpose of this paper is to investigate the effect of various mechanisms behind irreversible shrinkage, the observed trends are largely independent of the specific model utilized for $\mathrm{C}-\mathrm{S}-\mathrm{H}$. The irreversible shrinkage observed in Fig. 7 is a combined result of both mechanisms: cement grain dissolution/hydrate precipitation and $\mathrm{C}-\mathrm{S}-\mathrm{H}$ viscoplasticity. Comparing to the irreversible shrinkage occurring due to cement grain dissolution and hydrate precipitation effects, which is more significant at earlier ages, the relative importance of irreversible shrinkage caused by $\mathrm{C}-\mathrm{S}-\mathrm{H}$ viscoplasticity increases with age (through observing the differences between solid lines and dashed lines in Fig. 7 at different ages). This is due in part to the fact that the volume fraction of $\mathrm{C}-\mathrm{S}-\mathrm{H}$ phases inside cement paste increases with age. Without dramatic change in the mechanical properties of $\mathrm{C}-\mathrm{S}-\mathrm{H}$ phases (due to either $\mathrm{C}-\mathrm{S}-\mathrm{H}$ viscoplasticity or $\mathrm{C}-\mathrm{S}-\mathrm{H}$ aging), according to the simulation results, the hydration reaction (including combined effects of cement grain dissolution, hydrate precipitation and stiffness increase of cement paste) is the dominant mechanism leading to shrinkage irreversibility at early ages. The reason for this is that, over relatively short drying cycle periods (i.e., about 1 or 2 days), there is very little relaxation of the $\mathrm{C}-\mathrm{S}-\mathrm{H}$ given the retardation time and maximum relaxation used in the material model for $E(t)$. The material model chosen for $\mathrm{C}-\mathrm{S}-\mathrm{H}$ is not validated by experimental data for $\mathrm{C}-\mathrm{S}-\mathrm{H}$ given the lack of reliable data on $\mathrm{C}-\mathrm{S}-\mathrm{H}$ VE/VP properties. However, given the observed relaxation of cement paste (which includes both intrinsic $\mathrm{C}-\mathrm{S}-\mathrm{H}$ relaxation and dissolution effects) [48], it is reasonable to approximate early-age $\mathrm{C}-\mathrm{S}-\mathrm{H}$ relaxation according to the model used. Thus, the data in Fig. 7 indicate that, from a modeling perspective, it may be practicable to disregard irreversible shrinkage during daily drying cycles after the first drying.

In contrast to the limited significance of intrinsic $\mathrm{C}-$ S-H VP with respect to irreversible shrinkage during short-term drying cycles, Fig. 8 indicates that the 

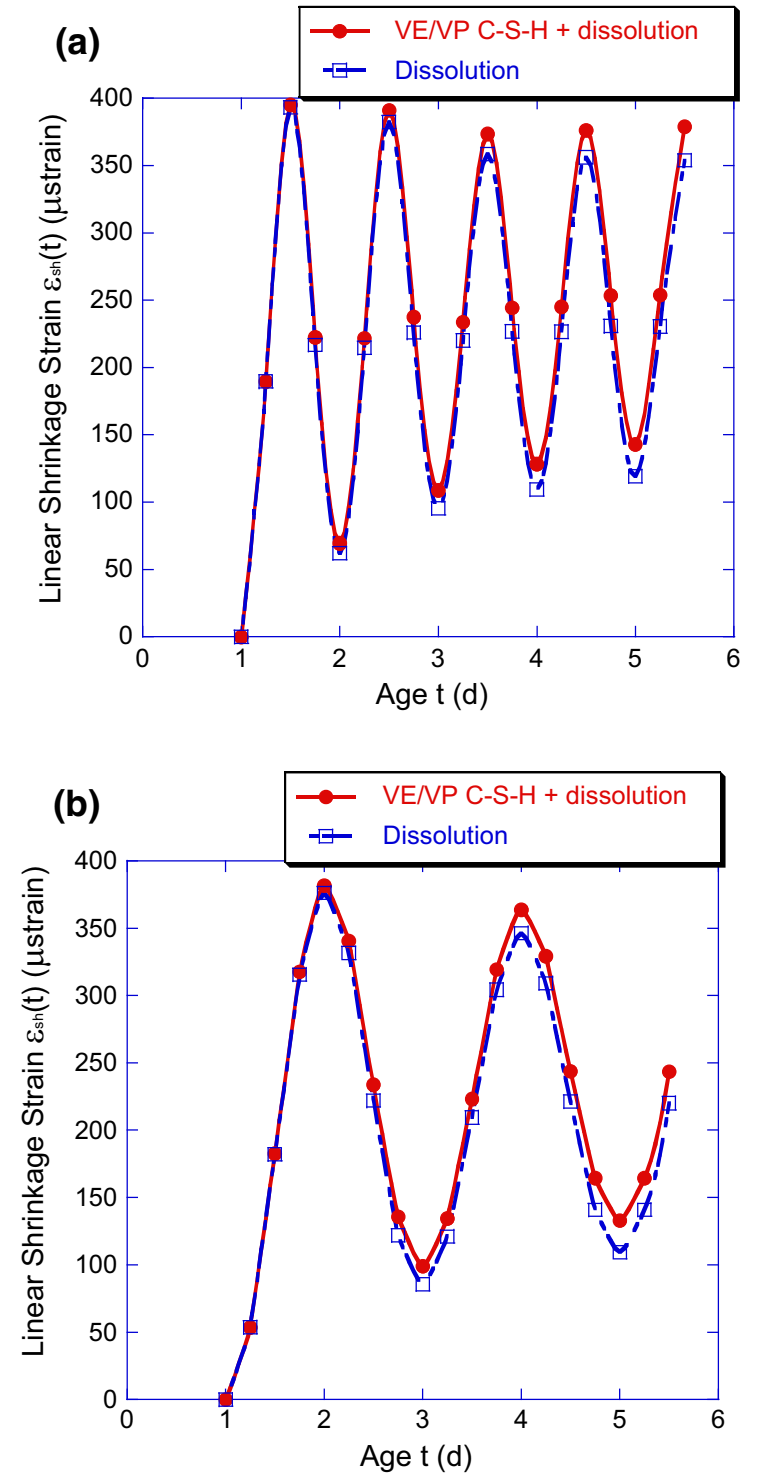

Fig. 7 Predicted shrinkage strain of $0.45 \mathrm{w} / \mathrm{c}$ cement paste when exposed to cyclic drying with different frequencies $\mathbf{a} f=1$ and $\mathbf{b} f=0.5$ when drying is initiated at an age of 1 day. All the lines in this figure show the overall shrinkage as a result of cyclic drying due to pore pressure change, cement grain dissolution/hydrate precipitation effects, and $\mathrm{C}-\mathrm{S}-\mathrm{H}$ timedependent behavior

influence of the intrinsic irreversible deformation is much more significant for long-term continuous desiccation. In Fig. 8, the same VE/VP Young's modulus and the Poisson's ratio of $\mathrm{C}-\mathrm{S}-\mathrm{H}$ was used as in Fig. 7. An assumed RH history $\mathrm{RH}(t)=$ $80+20 \exp (-t / 14)(\%)$ was applied at different initial drying ages of 1 and 14 days, where $t$ represents

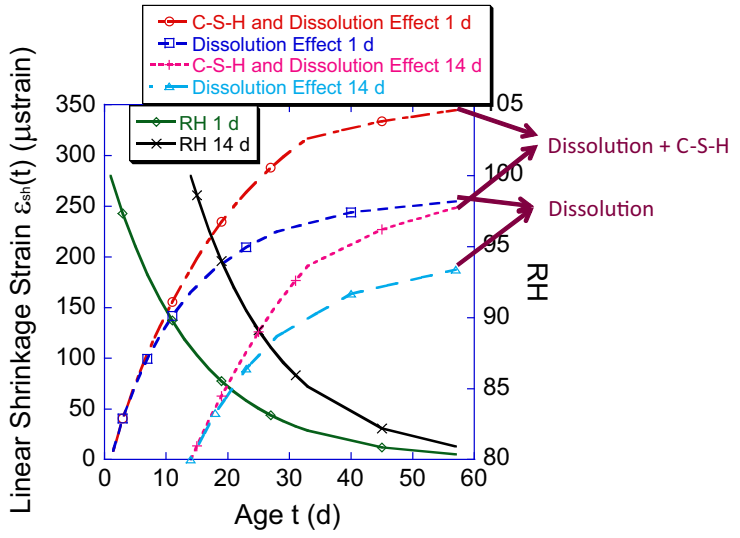

Fig. 8 Simulation of response of $0.40 \mathrm{w} / \mathrm{c}$ cement paste to longterm drying with ages of drying initiation of 1 and 14 days. The longer term drying allows more time for VP deformation to develop within the $\mathrm{C}-\mathrm{S}-\mathrm{H}$ than occurs during short-term cycles. The solid lines show the RH history, and the dashed lines show the simulated time-dependent shrinkage of cement paste

the time in days after the start of drying. In Fig. 8, the solid lines show the RH history, and the dashed lines show the time-dependent shrinkage of cement paste. It can be seen that the longer term drying provides a greater duration for the irreversible VP deformation to develop in the $\mathrm{C}-\mathrm{S}-\mathrm{H}$, thus making this component of greater potential importance at later ages versus cement grain dissolution and hydrate precipitation effects. Thus, in contrast to daily cycles, it may not be practicable to ignore long-term irreversible deformation of $\mathrm{C}-\mathrm{S}-\mathrm{H}$ under drying induced stresses. Ultimately, accurate determination of the contribution of $\mathrm{C}-\mathrm{S}-\mathrm{H}$ VP deformation to long-term irreversible desiccation shrinkage (associated with either cyclical or asymptotic drying) requires greater confidence in the VE/VP constitutive function assigned to $\mathrm{C}-\mathrm{S}-\mathrm{H}$, which requires a significant improvement in available experimental data.

\section{Conclusions}

To evaluate the mechanisms behind the irreversible desiccation shrinkage of cement paste at early ages, a computational model that couples a hydration microstructure evolution model and a finite element calculation routine was utilized to give predictions of the time-evolving shrinkage strain of cement paste. Virtual experiments were carried out, and based on the simulation results, dissolution of load-bearing cement grains 
and precipitation of hydrates that occurs during the hydration process appears to be a significant mechanism leading to irreversible shrinkage strain. Through the comparisons between the computationally predicted results with the results calculated through poroelasticity, it was concluded that poroelasticity likely underpredicts the overall early age shrinkage of cement paste as it neglects the cement grain dissolution and hydrate precipitation effect. The simulations also imply that irreversible shrinkage is closely related to age; delayed drying would lead to drastic reductions in irreversible shrinkage associated with cement grain dissolution and hydrate precipitation. According to cyclic drying and rewetting simulations, the number of cycles has a much smaller influence on the irreversible shrinkage than the age of the sample; thus, if drying cycles are sufficiently slow, then one would expect irreversible shrinkage only on the first cycle, whereas for fast cycles, one would expect irreversible shrinkage on subsequent cycles as well.

At early ages, simulation results indicate that the stress redistribution associated with the hydration reaction (including cement grain dissolution, hydrate precipitation and stiffness increase of cement paste) is significant with regards to irreversible desiccation shrinkage. For long term asymptotic drying, however, irreversible deformation within the $\mathrm{C}-\mathrm{S}-\mathrm{H}$ phase may also significantly impact irreversible shrinkage. It is impossible to determine the precise magnitude of influence at this time due to a lack of quality data for intrinsic, long-term $\mathrm{C}-\mathrm{S}-\mathrm{H}$ viscoplastic properties.

The primary limitation of the modeling approach described herein is the neglecting of the coupling between the hydration reaction and the internal $\mathrm{RH}$. Once the internal RH is reduced sufficiently, the hydration rate is substantially slowed or even halted (due to either kinetic or thermodynamic constraints). If hydration slows, then the dissolution of cement grains likely slows correspondingly, which would reduce the amount of irreversible shrinkage expected. However, at early ages where hydration reaction rates are more significant, the internal RH is generally not reduced to the point where the hydration rate would be significantly affected.

Acknowledgements This research was supported by the National Science Foundation under Grant numbers 0843979 and 1327314. Any opinions, findings, and conclusions or recommendations expressed in this material are those of the author(s) and do not necessarily reflect the views of the National Science Foundation.

\section{References}

1. Grasley ZC, Leung CK (2011) Desiccation shrinkage of cementitious materials as an aging, poroviscoelastic response. Cem Concr Res 41:77-89

2. Hansen W (1987) Drying shrinkage mechanisms in Portland cement paste. J Am Ceram Soc 70:323-328

3. Mindess S, Young JF, Darwin D (2002) Concrete, 2nd edn. Prentice Hall, Upper Saddle River

4. Feldman R, Sereda P (1970) A new model for hydrated Portland cement and its practical implications. Eng J 53:53-59

5. Feldman RF, Sereda PJ (1968) A model for hydrated Portland cement paste as deduced from sorption-length change and mechanical properties. Mater Struct 1:509-520

6. Powers TC (1965) Mechanisms of shrinkage and reversible creep of hardened cement paste. In: Brooks AE, Newman K (eds) The structure of concrete and its behaviour under load. Cement and Concrete Association, London, pp 319-344

7. Powers TC (1968) The thermodynamics of volume change and creep. Mater Struct 1:487-507

8. Young J, Berger R, Bentur A (1978) Shrinkage of tricalcium silicate pastes: superposition of several mechanisms Il. Cemento 75:391-398

9. Lura P, Jensen OM, van Breugel K (2003) Autogenous shrinkage in high-performance cement paste: an evaluation of basic mechanisms. Cem Concr Res 33:223-232. doi:10. 1016/S0008-8846(02)00890-6

10. Meyers BL, Slate FO (1970) Creep and creep recovery of plain concrete as influenced by moisture conditions and associated variables. Mag Concr Res 22:37-41

11. Bažant ZP (1972) Thermodynamics of hindered adsorption and its implications for hardened cement paste and concrete. Cem Concr Res 2:1-16. doi:10.1016/0008-8846(72)90019-1

12. Bentur A, Berger RL, Lawrence FV Jr, Milestone NB, Mindess S, Young JF (1979) Creep and drying shrinkage of calcium silicate pastes III. A hypothesis of irreversible strains. Cem Concr Res 9:83-95. doi:10.1016/00088846(79)90098-X

13. Bentur A, Milestone N, Young J (1978) Creep and drying shrinkage of calcium silicate pastes II. induced microstructural and chemical changes. Cem Concr Res 8:721-732

14. Helmuth RA, Turk DH (1967) Reversible and irreversible drying shrinkage of hardened portland cement and tricalcium silicate pastes. Portland Cem Assoc Res Dev Lab 9:8-21

15. Bullard JW, Lothenbach B, Stutzman PE, Snyder KA (2011) Coupling thermodynamics and digital image models to simulate hydration and microstructure development of portland cement pastes. J Mater Res 26:609-622

16. Lothenbach B, Winnefeld F (2006) Thermodynamic modelling of the hydration of portland cement. Cem Concr Res 36:209-226

17. Li X, Grasley ZC, Garboczi EJ, Bullard JW (2015) Computing the time evolution of the apparent viscoelastic/ 
viscoplastic Poisson's ratio of hydrating cement paste. Cement Concr Compos 56:121-133

18. Li X, Grasley ZC, Garboczi EJ, Bullard JW (2015) Modeling the apparent and intrinsic viscoelastic relaxation of hydrating cement paste. Cement Concr Compos 55:322-330

19. Li X, Rahman S, Grasley ZC (2016) Computationally implemented modeling of creep of composite materials caused by phase dissolution. Comput Mater Sci 125:61-71. doi:10.1016/j.commatsci.2016.08.030

20. Pignatelli I, Kumar A, Alizadeh R, Le Pape Y, Bauchy M, Sant G (2016) A dissolution-precipitation mechanism is at the origin of concrete creep in moist environments. J Chem Phys 145:054701. doi:10.1063/1.4955429

21. Bazant ZP, Chern JC (1985) Concrete creep at variable humidity: constitutive law and mechanism. Mater Struct 18:1-20. doi:10.1007/bf02473360

22. Jennings HM, Kumar A, Sant G (2015) Quantitative discrimination of the nano-pore-structure of cement paste during drying: new insights from water sorption isotherms. Cem Concr Res 76:27-36. doi:10.1016/j.cemconres.2015. 05.006

23. Sorelli L, Frech-Baronet J, Charron J-P (2015) Creep behavior of cement paste, mortar, and concrete: the role of relative humidity and interface porosity. In: CONCREEP 10. pp 296-305. doi:10.1061/9780784479346.034

24. Wittmann F (1970) Einfluß des Feuchtigkeitsgehaltes auf das Kriechen des Zementsteines. Rheol Acta 9:282-287. doi: $10.1007 / \mathrm{bf0} 1973489$

25. Li X, Grasley ZC (2015) Shrinkage and creep caused by dissolution. In: CONCREEP 10. pp 318-326. doi:10.1061/ 9780784479346.037

26. Parrot LJ, Killoh DC (1984) Prediction of cement hydration. In: Chemistry and Chemically-related Properties of Cement, London, Engl, 1984. British Ceramic Proceedings. British Ceramic Society, pp 41-53

27. Kulik DA (2002) Gibbs energy minimization approach to modeling sorption equilibria at the mineral-water interface: thermodynamic relations for multi-site-surface complexation. Am J Sci 302:227-279. doi:10.2475/ajs.302.3.227

28. Kulik DA (2006) Dual-thermodynamic estimation of stoichiometry and stability of solid solution end members in aqueous-solid solution systems. Chem Geol 225:189-212. doi:10.1016/j.chemgeo.2005.08.014

29. Ruetz W (1968) A hypothesis for the creep of hardened cement paste and the influence of simultaneous shrinkage. In: Proceedings of international conference on the structure of concrete. Cement and Concrete Association, London

30. Zohdi TI (2004) Homogenization methods and multiscale modeling. In: Encyclopedia of computational mechanics. Wiley, New York. doi:10.1002/0470091355.ecm034

31. Christensen R (2012) Theory of viscoelasticity: an introduction. Elsevier, New York

32. Wineman AS, Rajagopal KR (2000) Mechanical response of polymers: an introduction. Cambridge University Press, Cambridge

33. Bentz DP, Garboczi EJ, Quenard DA (1998) Modelling drying shrinkage in reconstructed porous materials: application to porous Vycor glass. Modell Simul Mater Sci Eng 6:211

34. Tanabe T-a, Sakata K, Mihashi H, Sato R, Maekawa K, Nakamura H (2008) Creep, shrinkage and durability mechanics of concrete and concrete structures. In: Two volume set: proceedings of the CONCREEP 8 conference held in Ise-Shima, Japan, 30 September-2 October 2008. CRC Press, Boca Raton

35. Haecker CJ, Garboczi EJ, Bullard JW, Bohn RB, Sun Z, Shah SP, Voigt T (2005) Modeling the linear elastic properties of Portland cement paste. Cem Concr Res 35:1948-1960. doi:10.1016/j.cemconres.2005.05.001

36. Garci Juenger MC, Jennings HM (2002) Examining the relationship between the microstructure of calcium silicate hydrate and drying shrinkage of cement pastes. Cem Concr Res 32:289-296. doi:10.1016/S0008-8846(01)00673-1

37. Di Bella C, Wyrzykowski M, Lura P (2016) Evaluation of the ultimate drying shrinkage of cement-based mortars with poroelastic models. Mater Struct 50:52. doi:10.1617/ s11527-016-0870-0

38. Oey $\mathrm{T}$ et al (2016) The influence of water activity on the hydration rate of tricalcium silicate. J Am Ceram Soc 99:2481-2492. doi:10.1111/jace.14181

39. Grasley ZC, Rajagopal KR (2011) Revisiting total, matric, and osmotic suction in partially saturated geomaterials. Z Angew Math Phys 63:373-394. doi:10.1007/s00033-0110168-6

40. Defay R, Bellemans A, Prigogine I (1966) Surface tension and adsorption. Longmans, London

41. Grasley ZC (2006) Measuring and modeling the time-dependent response of cementitious materials to internal stresses. University of Illinois at Urbana-Champaign, Champaign

42. Jensen OM, Hansen PF, Lachowski EE, Glasser FP (1999) Clinker mineral hydration at reduced relative humidities. Cem Concr Res 29:1505-1512. doi:10.1016/S00088846(99)00132-5

43. Wyrzykowski M, Lura P (2016) Effect of relative humidity decrease due to self-desiccation on the hydration kinetics of cement. Cem Concr Res 85:75-81. doi:10.1016/j. cemconres.2016.04.003

44. Hajibabaee A, Grasley Z, Ley MT (2016) Mechanisms of dimensional instability caused by differential drying in wet cured cement paste. Cem Concr Res 79:151-158. doi:10. 1016/j.cemconres.2015.09.007

45. Pinson MB et al (2015) Hysteresis from multiscale porosity: modeling water sorption and shrinkage in cement paste. Phys Rev Appl 3:064009

46. Grasley Z, El-Helou R, D’Ambrosia MD, Mokarem D, Moen C, Rajagopal KR (2015) Model of infinitesimal, nonlinear elastic response of concrete subjected to uniaxial compression. J Eng Mech 141:04015008

47. Neville AM (1995) Properties of concrete. Pearson Education Ltd., London

48. Galitz C, Grasley Z (2015) Effects of strain reversal on elastic and viscoelastic properties of early age cement paste beams. Mater Struct 49(4):1-13. doi:10.1617/s11527-0150576-8 